\title{
Research and Application of Optimal Combination of Quick Drop Valve Flow Regenerative Performance Based on Orthogonal Experimental Design
}

\author{
Xiaoxiang $\mathrm{Li}^{1, \mathrm{a}}$, Anlin Wang ${ }^{1}$ and Jiadong $\mathrm{Lin}^{2}$ \\ ${ }^{1}$ School of Mechanical Engineering, Tongji University, 4800 Caoan Highway, Shanghai, China \\ ${ }^{2}$ Research \& Development Center, Shantui Construction Machinery Co.,Ltd, No. 58, Highway G327, Jining, \\ Shandong, China
}

\begin{abstract}
Based on the simulation model of quick drop valve flow regeneration measurement data, the influence factors of quick drop valve are analysed through two orthogonal experimental designs to obtain the optimal combination of the efficiency of oil filled $\beta$ for flow regeneration. Simulation and experimental verification results show that: In the structure of the original quick drop valve, through the range $\mathrm{R}$, the influence factor valve opening $\mathrm{C}$ has the greatest influence on the efficiency of oil filled $\beta$. The efficiency of oil filled $\beta$ of the optimal combination $\mathrm{A}_{1} \mathrm{~A}_{2} \mathrm{~A}_{3}$ is $78.9 \%$ lower than the standard requirement of $85 \%$. Based on the method of controlling the oil return variable according to the load feedback and orthogonal experimental design, the improved structure makes the efficiency of oil filled $\beta$ for flow regeneration increase from $57 \%$ of the measured data to more than $85 \%$ of the measured verification. Through 1000 hours performance verification, the performance of the quick drop valve is stable, no failure rate, and the efficiency of oil filled $\beta$ is still higher than $85 \%$. At the same time, the efficiency of the earth-moving work after the quick drop of the blade is increased by more than $28 \%$.
\end{abstract}

\section{Introduction}

In some construction machinery such as bulldozer blades lowering process, as one of the important hydraulic components of the hydraulic system, the quick drop valve can connect the rod-less cavity and the rod cavity of the hydraulic cylinder, reduce the flow of the oil return tank in the hydraulic cylinder, thereby shortening the time lag before the blade starts to dig. The flow regeneration performance of the quick drop valve has an important influence on the operation efficiency, and at the same time, its function can make the blade lower quickly to complete the freezing operation of some frozen soils in extreme cold conditions. At present, there is very little information on the theoretical mechanism of the flow regeneration of the quick drop valve. Most of the information is the introduction to the principle of its function.

The principle of paralleling the hydraulic pipe in the quick drop valve pipe to increase the blade's lowering speed was elaborated[1]. However, the flow regeneration performance of the quick drop valve had not been researched from the theoretical mechanism. Kong only tested the blade lowering

\footnotetext{
${ }^{\text {a }}$ Corresponding author : lixiaoxiang@tongji.edu.cn
} 
time with or without a quick drop valve in bulldozer's working hydraulic system[2]. Wang and Tang introduced the function and principle of quick drop valve for loader, and did not analyze the performance theoretically[3]. Li et al introduced the failure of the quick drop valve during the lifting of the bulldozer blade[4-8]. Simple analysis of the selection of the return spring in the quick drop valve[9].The author had theoretically analyzed the influence degree of multi-valve flow characteristic coefficient on flow regeneration performance in working hydraulic system and did not consider the performance of the quick drop valve[10].

Based on the analysis of the influence of two orthogonal test designs on the influence factors of the quick drop valve, the improvement of the structure is achieved by using the method of load feedback to control the return oil variable. The efficiency of oil filled $\beta$ of the quick drop valve is increased from $57 \%$ of the measured data to $85 \%$ of the measured verification, and the efficiency of the earthmoving work after lowering quickly is increased by more than $28 \%$. The quick drop valve is verified by a failure-free performance test of more than 1000 hours.

\section{Modeling and calibration}
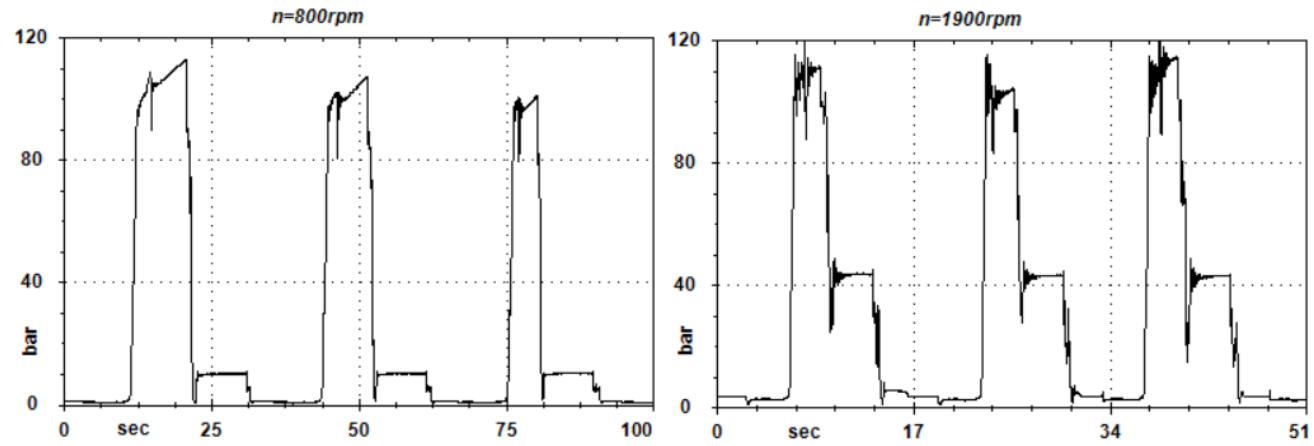

Figure 1. Pressure curve of rod-less cavity of cylinder

The performance test of the quick drop valve is based on the engine speed $n=800 \mathrm{rpm}$ and $n=1900 \mathrm{rpm}$ of a certain type of bulldozer. The pressure curve of rod-less cavity of cylinder is shown in Figure 1. According to the Figure 1, It can be seen that during the process of the blade lowering quickly: $t=7.8 \mathrm{~s}$ for $n=800 \mathrm{rpm}$, and $\mathrm{t}=3.7 \mathrm{~s}$ for $\mathrm{n}=1900 \mathrm{rpm}$. Calculated by the cylinder stroke and the outlet flow rate of the quick drop valve, the efficiency of oil filled $\beta$ are $56.6 \%$ and $56.9 \%$ respectively. It is known that the engine speed has little effect on the flow regeneration performance of the quick drop valve.

The system simulation software Simulation X is used to model the system and the system model is calibrated by the measured value. The pressure curve of rod-less cavity of cylinder of the hydraulic system simulation model is shown in Figure 2. Based on the simulation curves of flow regeneration and pressure build-up time, the efficiency of oil filled $\beta$ of the flow regeneration is calculated. The efficiency of oil filled $\beta$ at the engine speed is $56.7 \%$ and $57 \%$ respectively, and the error between simulation and measurement is less than $0.5 \%$. It can be considered that the simulation results of the system model have a high consistency with the measured data.
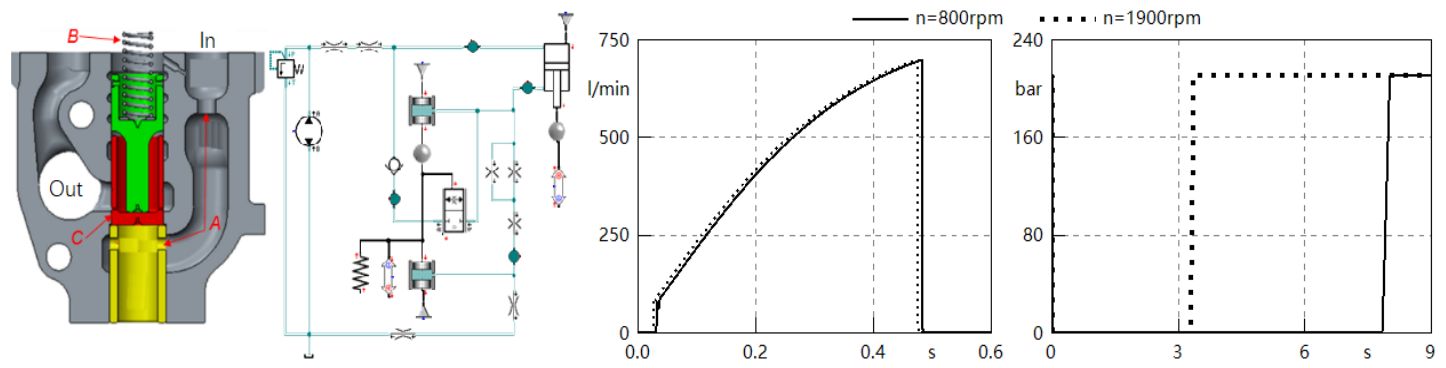

Figure 2. System simulation model, curves of flow regeneration and pressure build-up time 


\section{Orthogonal experimental design}

It can be seen from the structure diagram of the quick drop valve in Figure 2.The flow regeneration performance of the quick drop valve is related to the structural parameters such as the orifice A, the return spring stiffness $\mathrm{B}$ and the valve opening $\mathrm{C}$. The Orthogonal experimental design is used to analyze the influence of the above three parameters on flow regeneration and the optimal level combination. Considering factors such as pressure loss, spool leakage requirement and space structure limitation, the selected parameters value are shown in Table 1.

Table 1. Parameter value table

\begin{tabular}{|c|c|c|c|c|c|c|}
\hline Parameter & \multicolumn{2}{|c|}{$A_{1}$} & \multicolumn{2}{c|}{$A_{2}$} & \multicolumn{2}{c|}{$A_{3}$} \\
\hline Value & $2-\Phi 8 \mathrm{~mm}+\Phi 13 \mathrm{~mm}$ & \multicolumn{2}{|c|}{ 2- $\Phi 10 \mathrm{~mm}+\Phi 13 \mathrm{~mm}$} & \multicolumn{2}{|c|}{ 2- $\Phi 12 \mathrm{~mm}+\Phi 13 \mathrm{~mm}$} \\
\hline Parameter & $B_{1}$ & $B_{2}$ & $B_{3}$ & $C_{1}$ & $C_{2}$ & $C_{3}$ \\
\hline Value & $3 \mathrm{~N} / \mathrm{mm}$ & $5 \mathrm{~N} / \mathrm{mm}$ & $7 \mathrm{~N} / \mathrm{mm}$ & $7 \mathrm{~mm}$ & $8 \mathrm{~mm}$ & $9 \mathrm{~mm}$ \\
\hline
\end{tabular}

According to the L9-orthogonal table, 9 orthogonal experimental designs are performed. The data is shown in Table 2. According to the $K_{i}(i=1,2,3)$ value, the optimal level combination is, and the corresponding the efficiency of oil filled $\beta$ for flow regeneration is $78.9 \%$. It can be seen from the range $\mathrm{R}$ that the structural parameter of the valve opening $\mathrm{C}$ has the greatest influence on the efficiency of oil filled $\beta$. There is still a certain gap between the efficiency of oil filled $\beta$ and the standard requirement of $85 \%$ of the quick drop valve under the optimal level combination condition.

Table 2. Efficiency of oil filled $\beta$ experimental data and calculation

\begin{tabular}{|c|c|c|c|c|c|}
\hline \multirow{4}{*}{$\begin{array}{l}\text { Experiment } \\
\text { number }\end{array}$} & \multicolumn{5}{|c|}{ Factors } \\
\hline & $\begin{array}{c}\text { Orifice } \\
\text { A }\end{array}$ & $\begin{array}{c}\text { Return spring } \\
\text { stiffness B }\end{array}$ & $\begin{array}{c}\text { Valve opening } \\
\text { C }\end{array}$ & & Experiment result \\
\hline & \multicolumn{5}{|c|}{ Level } \\
\hline & 1 & 2 & 3 & 4 & Efficiency of oil filled $\beta(\%)$ \\
\hline 1 & 1 & 1 & 1 & 1 & 78.9 \\
\hline 2 & 1 & 2 & 2 & 2 & 71.4 \\
\hline 3 & 1 & 3 & 3 & 3 & 57 \\
\hline 4 & 2 & 1 & 2 & 3 & 66.2 \\
\hline 5 & 2 & 2 & 3 & 1 & 52.2 \\
\hline 6 & 2 & 3 & 1 & 2 & 74.3 \\
\hline 7 & 3 & 1 & 3 & 2 & 49.1 \\
\hline 8 & 3 & 2 & 1 & 3 & 71.9 \\
\hline 9 & 3 & 3 & 2 & 1 & 63 \\
\hline$K_{I}$ & 69.1 & 64.8 & 75.1 & & \\
\hline$K_{2}$ & 64.3 & 62.2 & 66.9 & & $\mathrm{~T}=584$ \\
\hline$K_{3}$ & 61.3 & 64.7 & 52.7 & & \\
\hline$R$ & 7.8 & 2.6 & 22.4 & & \\
\hline
\end{tabular}

It is difficult to increase the efficiency of oil filled $\beta$ on the basis of the current limit of the valve opening and the space structure. Taking the first two items in the influence factor that have a large influence on the efficiency of oil filled $\beta$ as the research object, the disadvantages of the quantitative feedback structure of the orifice A and the separated structure of the valve spool are analyzed. The method of variable control based on feedback from the load and the integrated structure of the valve spool structure are used to improve the performance of the quick drop valve. The following section will model and simulate the flow regeneration performance of the quick drop valve with improved structure and analyze orthogonal experimental data. 


\section{Improve performance}

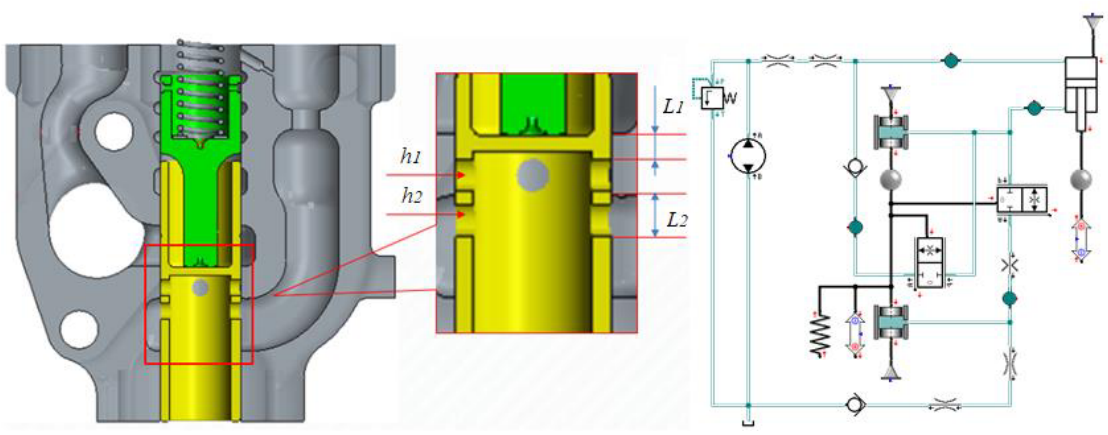

Figure 3. Improved quick drop valve structure diagram and system simulation model

Based on the analysis in the previous section, the structure of the quick drop valve is improved and a simulation model is established. As shown in Figure 3, the parameters of the variables are $h_{1}, h_{2}, L_{1}$, and $L_{2}$. Under the conditions of pressure loss, spool leakage requirement and space structure limitation, the Orthogonal experimental design is used to analyze the influence of the above four parameters on flow regeneration and the optimal level combination. The specific parameters are shown in Table 3.

Table 3. Parameter value table

\begin{tabular}{|c|c|c|c|c|c|c|c|c|c|c|c|c|}
\hline Parameter & $h_{11}$ & $h_{12}$ & $h_{13}$ & $h_{21}$ & $h_{22}$ & $h_{23}$ & $L_{11}$ & $L_{12}$ & $L_{13}$ & $L_{21}$ & $L_{22}$ & $L_{23}$ \\
\hline Value $(\mathrm{mm})$ & 4- $\Phi 8$ & 5- $\Phi 8$ & 6- $\Phi 8$ & 2- $\Phi 8$ & 2- $\Phi 9$ & 2- $\Phi 10$ & 3 & 4 & 5 & 12 & 15 & 18 \\
\hline
\end{tabular}

According to the L9-orthogonal table, 9 orthogonal experimental designs are performed. The data is shown in Table 4. According to the $K_{i}(i=1,2,3)$ value, the optimal level combination is $h_{13} h_{21} L_{11} L_{21}$, and corresponding the efficiency of oil filled $\beta$ for flow regeneration is $94.3 \%$ which meets the standard requirement of $85 \%$. It can be seen from the range $\mathrm{R}$ that the influence factor under-lap $L_{2}$ has the greatest influence on the efficiency of oil filled $\beta$. Considering the orifice $h_{l}$ from the layout of the valve spool structure, $h_{11}$ is the best. Considering the orifice $h_{2}$ from the pressure loss, $h_{23}$ is the best. Considering the amount of valve spool leakage from over-lap $L_{1}, L_{13}$ is the best. Since the influence factor under-lap $L_{2}$ has the greatest influence on the efficiency of oil filled $\beta$, the parameter $L_{2}$ is adjusted based on $h_{11} h_{23} L_{13}$.

Table 4. Efficiency of oil filled $\beta$ experimental data and calculation

\begin{tabular}{|c|c|c|c|c|c|}
\hline \multirow{3}{*}{$\begin{array}{c}\text { Experiment } \\
\text { number }\end{array}$} & \multicolumn{5}{|c|}{ Factors } \\
\cline { 2 - 6 } & Orifice $h_{1}$ & Orifice $h_{2}$ & Over-lap $L_{1}$ & Under-lap $L_{2}$ & Experiment result \\
\cline { 2 - 6 } & 1 & 2 & 3 & 4 & Efficiency of oil filled $\beta(\%)$ \\
\hline 1 & 1 & 1 & 1 & 1 & 92.3 \\
2 & 1 & 2 & 2 & 2 & 74 \\
3 & 1 & 3 & 3 & 3 & 61.1 \\
4 & 2 & 1 & 2 & 3 & 73 \\
5 & 2 & 2 & 3 & 1 & 91.3 \\
6 & 2 & 3 & 1 & 2 & 78.9 \\
7 & 3 & 1 & 3 & 2 & 76.9 \\
8 & 3 & 2 & 1 & 3 & 93 \\
9 & 3 & 3 & 2 & 1 & T=719.2 \\
\hline$K_{1}$ & 75.8 & 81.4 & 82.6 & 92.2 & \\
$K_{2}$ & 81.1 & 80.7 & 80 & 77.3 & \\
$K_{3}$ & 82.9 & 77.7 & 76.4 & 75.7 & \\
\hline$R$ & 7.1 & 3.7 & 6.2 & 16.5 & \\
\hline
\end{tabular}


Simulation analysis is performed in the range of $12 \mathrm{~mm} \leq L_{2} \leq 24 \mathrm{~mm}$, and the efficiency of oil filled $\beta$ is shown in Table 5. With the increase size of $L_{2}$, the efficiency of oil filled $\beta$ gradually decreases and tends to be stable, and the decreasing amplitude $\Delta \beta$ gradually decreases. Take $L_{2}$ as $12 \mathrm{~mm}\left(L_{2}=\right.$ $12.5 \mathrm{~mm}, \beta=84.9 \%$ ) and use the parameter combination $h_{11} h_{23} L_{13} L_{21}$ to improve the structure. The following section will verify the improved performance of the quick drop valve.

Table 5. Efficiency of oil filled $\beta$ at $12 \mathrm{~mm} \leq L_{2} \leq 24 \mathrm{~mm}$

\begin{tabular}{|c|c|c|c|c|c|c|c|c|c|c|c|c|}
\hline Value $(\mathrm{mm})$ & 12 & 13 & 14 & 15 & 17 & 18 & 19 & 20 & 21 & 22 & 23 & 24 \\
\hline$\beta(\%)$ & 89 & 81 & 74.3 & 69.3 & 65.6 & 63 & 61.2 & 60.1 & 59.4 & 59.3 & 59.3 & 59.3 \\
\hline$\Delta \beta(\%)$ & & 8 & 6.7 & 5 & 3.7 & 2.6 & 1.8 & 1.1 & 0.7 & 0.1 & 0 & 0 \\
\hline
\end{tabular}

\section{Verification}

Based on the analysis in the previous section, the structure of the quick drop valve is improved with $h_{11} h_{23} L_{13} L_{21}$. The performance of the quick drop valve is also tested on the basis of the same bulldozer engine speed $n=800 \mathrm{rpm}$ and $n=1900 \mathrm{rpm}$. The improved quick drop valve and the pressure curve of the tested rod-less cavity of cylinder are shown in Figure 4. It can be seen that during the process of the blade lowering quickly, $\mathrm{t}=5.55 \mathrm{~s}$ for $\mathrm{n}=800 \mathrm{rpm}$, and $\mathrm{t}=2.36 \mathrm{~s}$ for $\mathrm{n}=1900 \mathrm{rpm}$. Calculated by the relevant data, the efficiency of oil filled $\beta$ are $87.9 \%$ and $88.1 \%$ respectively. The error between the test data and the simulation theory data is about $1 \%$. Considering the errors in parts manufacturing and testing, the results meet the requirements.
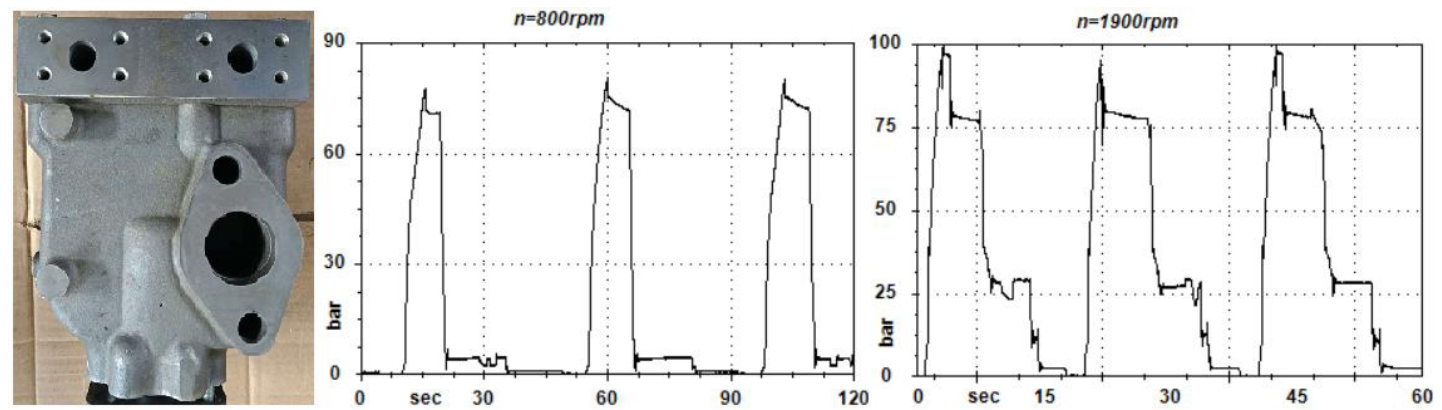

Figure 4. The improved quick drop valve and the pressure curve of the tested rod-less cavity of cylinder

So far, the quick drop valve has passed the verification of more than 1000 hours, and the performance is stable and no failure. The efficiency of oil filled $\beta$ is still higher than $85 \%$. The improvement of quick drop valve performance can effectively solve the problem that the efficiency of oil filled $\beta$ is lower than the standard requirement of $85 \%$.

\section{Conclusions and future work}

Through the theoretical analysis and verification of the flow regeneration performance of the quick drop valve, the following conclusions can be drawn:

(1) In the structure of the original quick drop valve, the influence factor valve opening $C$ has the greatest influence on the efficiency of oil filled $\beta$, the optimal level combination is $A_{1} B_{l} C_{1}$, and the efficiency of oil filled $\beta=78.9 \%$ is lower than the standard requirement of $85 \%$.

(2) In the improved structure, the influence factor under-lap $L_{2}$ has the greatest influence on the efficiency of oil filled $\beta$, the optimal level combination is $h_{11} h_{23} L_{13} L_{21}$, and the corresponding efficiency of oil filled $\beta=94.3 \%$ is higher than the standard requirement of $85 \%$.

(3) Considering the layout of the spool structure, pressure loss and the spool leakage requirement, we finally use the parameter combination $h_{11} h_{23} L_{13} L_{21}$ to improve the structure. 
(4) The structural improvement based on the method of controlling the oil return variable according to the load feedback makes the quick drop valve efficiency of oil filled $\beta$ for flow regeneration increase from $57 \%$ of the measured data to more than $85 \%$ of the measured verification, and the efficiency of the earth-moving work after lowering quickly is increased by more than $28 \%$.

We propose, and are currently involved in, the following extensions of the current work. First, consider the influence of the flow regeneration function of the quick drop valve on the performance of the working hydraulic system from the aspect of matching the entire machine power performance. Second, the feedback control of the flow regeneration function of the quick drop valve should be changed from mechanical to electrical. Real-time detection and tracking of pressure changes when the blade is lowering quickly, forming an effective closed-loop control system.

\section{References}

1. Y.S. Jiang, L.L. Song, K. Jin, J.J. Zhao, J. Wang, A.L. Lv, C. Mechanization, 2, 57(2013)

2. L.P. Kong, Y.X. Gao, C. Mechanization, 10, 36(2017)

3. D.P. Wang, R.X. Tang, H. Lin, Y.W. Feng, Mach Tool Hydrau, 44,89 (2016)

4. Q.Y. Li, J.B. Song, J.M. Ding, X.L. Guan, W.Z. Zhang, C.MACHINERY, 9, 104, (2013)

5. L.X. Geng, J.M. Gao, CM\&M 9,146, (2014)

6. C.P. Chen, CM\&E, 33,50, (2002)

7. N. Yang, CM\&M, 5,160, (2007)

8. Y.P. Tian, CM\&M, 5,159, (2005)

9. C.L. Li, X.X. Song, CM\&E, 2,14, (1987)

10. X.X. Li, G.P. Li, S.B. Wang, P.Hu, Chin Hydrau Pneumatics, 9, 49(2009) 